\title{
Nutrição materna e programação fetal: o papel dos hábitos alimentares no desenvolvimento embrionário e pós-natal
}

\author{
Maternal nutrition and fetal programming: \\ The role of food in pre-and post-natal development
}

Carolina Bellato de Souza CAMPOS $^{1}$

Adrianne Christine Palanch ${ }^{\mathrm{II}}$

${ }^{\mathrm{I}}$ Universidade Metodista de Piracicaba (UNIMEP), Piracicaba/ SP - Brasil

"Universidade Metodista de Piracicaba (UNIMEP), Piracicaba/ SP - Brasil
RESUMo A incidência de doenças cardiovasculares e obesidade está associada ao aumento da ingestão de alimentos hipercalóricos em decorrência de modificações no padrão alimentar ocorrido nas últimas décadas. A dieta hiperlipídica administrada a gestantes induz, nos descendentes, aumento da pressão sanguínea, resistência à insulina, dislipidemia e obesidade. Esses são sintomas característicos da Síndrome Metabólica que estão sendo descritos cada vez mais em crianças e adolescentes. Neste trabalho, foram analisadas informações obtidas dos bancos de dados bibliográficos (SCIELO, MEDLINE, PUBMED) enfocando pesquisas sobre nutrição materna durante a gravidez e lactação e a influência desses fatores nutricionais no desenvolvimento dos conceptos. Para isso, verificamos artigos científicos e livros acadêmicos principalmente dos últimos dez anos tirados de revistas nacionais e internacionais, assim como, do Ministério da Saúde do Brasil. As informações obtidas com este trabalho permitem observar a incidência de alterações metabólicas em parturientes e sua relação com a saúde dos seus descendentes. Dessa forma, será possível sugerir uma relação entre o estado de saúde e a nutrição materna com o desenvolvimento fetal e características fisiológicas no recém-nascido.

Palavras-chave: Dieta materna. Lactação. Programação fetal. Subnutrição E ObEsidade.

Aвstract In the last decades, the incidence of cardiovascular disease and obesity is associated with increased intake of high-calorie foods as a result of changes in dietary patterns. A high fat diet administered to pregnant women induces in the offspring increased blood pressure, insulin resistance, dyslipidemia and obesity. These are typical symptoms of Metabolic Syndrome being described increasingly in children and teenagers. In this research was analyzed data from library, such as, Scielo, Medline, PubMed, with focus in maternal nutrition during pregnant and lactating and the influence of this nutritional factors in offspring development. With this objective, we studied mainly the last 10 years publications in scientific articles and academics books, including the Brazilian Health Department. The information obtained from this research allow observe the incidence of metabolic disorders in mothers and their relation to the health of their offspring. Thus, it is possible to suggest a relationship between health status and maternal nutrition with fetal development and physiological characteristics in newborns.

Key-words: Maternal diet. Lactation. Fetal programming. SUBNUTRITION AND OBESITY. 


\section{INTRODUÇÃO}

As últimas décadas foram marcadas por mudanças no estilo de vida que tem afetado a saúde das pessoas. Dietas hipercalóricas aliadas ao sedentarismo alteram a dinâmica energética do organismo. Mais de 90\% da população não consome a quantidade recomendada de frutas, verduras e legumes, sendo alto o consumo de bebidas açucaradas, alimentos calóricos e sódio em grande quantidade. $^{1,2}$

A dieta materna pode exercer influência direta nos filhos. A restrição do crescimento intrauterino e o baixo peso ao nascer, acompanhados de ganho de peso excessivo na infância e na adolescência, estão associados à obesidade, resistência insulínica, cardiopatias, hipertensão arterial, hiperfagia entre outras, caracterizando um quadro de Síndrome Metabólica. ${ }^{1,3,4,5,6}$

O termo "Programação Fetal" foi utilizado por Hales e Barker ${ }^{4}$ para demonstrar que doenças metabólicas têm origem na gravidez. Qualquer estímulo nutricional ou estresse durante o desenvolvimento intrauterino resultam em respostas adaptativas do feto, que são vantajosas para a sua sobrevivência em um ambiente com condições abaixo do ideal. Entretanto, essas adaptações tornam-se desfavoráveis em aspectos anatômicos, metabólicos e fisiológicos, predispondo o feto à Síndrome Metabólica nas fases pós-nascimento. ${ }^{7}$

\section{MÉTOdos}

Para a elaboração desta revisão bibliográfica foi realizado levantamento de informações científicas a partir de livros, artigos, teses e periódicos da área com as bases de acesso Medline, Scielo, PubMed e Google Acadêmico. Sendo a pesquisa realizada em português e inglês, principalmente, no período compreendido entre os últimos dez anos, exceto alguns artigos pioneiros sobre o tema, e utilizando os seguintes descritores: dieta materna, lactação, programação fetal, desnutrição, obesidade.

\section{Avaliação e ReCOMENDAÇões NUTRICIONAIS NA GESTAÇÃO}

Um dos principais fatores de prevenção da morbidade e da mortalidade perinatal, da promoção da saúde da mulher e de um prognóstico preciso da situação de saúde da criança, está relacionado ao acompanhamento nutricional durante a gestação. Na rede básica de saúde (SUS), a avaliação nutricional é realizada por antropometria (peso, altura e idade gestacional) no pré-natal por uma equipe multidisciplinar, segundo recomendações do Ministério da Saúde. ${ }^{8}$

A avaliação do consumo alimentar envolve fatores biológicos, socioeconômicos, culturais e ambientais, sendo mais complexa em gestantes, por apresentarem alterações fisiológicas e psicológicas. Durante a avaliação são identificados grupos propensos a distúrbios nutricionais que interferem na gestação e saúde do concepto. Como os relacionados ao consumo de ferro e à ocorrência de anemia ${ }^{9}$ que aumentam o risco de parto prematuro, baixo peso ao nascer, pré-eclâmpsia e aborto espontâneo. ${ }^{10}$

O excesso de ganho de peso materno e fetal pode estar associado a diabetes gestacional, levando à dificuldades no parto, enquanto que um baixo ganho de peso ges- 
tacional está relacionado a um aumento no risco de retardo de crescimento intrauterino e mortalidade perinatal. ${ }^{11}$

\section{HÁbitos ALIMENTARES dA GESTANTE}

Durante a gestação, o organismo necessita de um aporte diferenciado de nutrientes, para um adequado crescimento e desenvolvimento fetal, pois a nutrição do concepto vem das reservas nutricionais da mãe. ${ }^{6,12}$ Essas informações nutricionais da gestação estão resumidamente indicadas na Tabela 1.

Dependendo da idade materna, a necessidade nutricional pode ser diferenciada. Gestantes muito jovens não estão preparadas fisiologicamente para suportar o estresse da gravidez, e o seu ganho de peso durante a gestação é menor, podendo haver competição por nutrientes entre ela $\mathrm{e}$ o feto..$^{13}$ As adolescentes também estão em risco nutricional devido aos hábitos alimentares e excesso de atividade física. ${ }^{8}$

Tabela 1. Recomendação nutricional para gestantes

\begin{tabular}{|c|c|c|c|}
\hline Elemento & Função & $\begin{array}{c}\text { Recomendação } \\
\text { nutricional }\end{array}$ & Fonte nutricional \\
\hline Proteína & $\begin{array}{l}\text { Fonte de aminoácidos - } \\
\text { síntese proteica. }\end{array}$ & $\begin{array}{l}\text { 1,1g } / \mathrm{kg} / \mathrm{dia}^{14} \\
0,75 \text { a } 1,0 \mathrm{~g} / \mathrm{Kg} / \text { dia mais } 6 \\
\text { g/dia }{ }^{15}\end{array}$ & $\begin{array}{l}\text { Carnes, derivados } \\
\text { de leite, ovos, } \\
\text { leguminosas, } \\
\text { oleaginosas. }\end{array}$ \\
\hline Carboidrato & Fonte de energia. & $\begin{array}{l}175 \mathrm{~g} / \text { dia }^{14} \\
55 \text { a } 75 \% \text { do VET diário }\end{array}$ & $\begin{array}{l}\text { Cereais, frutas, } \\
\text { hortaliças, açúcar. }\end{array}$ \\
\hline Lipídio & $\begin{array}{l}\text { Fonte de energia, } \\
\text { metabolismo e síntese de } \\
\text { hormônios. }\end{array}$ & $\begin{array}{l}15 \text { a } 30 \% \text { total do VET, até } \\
10 \% \text { de gordura saturada } \\
\text { n- } 6-13 \text { g/dia e de n-3 1,4 } \\
\text { g/dia }{ }^{15}\end{array}$ & $\begin{array}{l}\text { Óleos vegetais e de } \\
\text { origem animal. }\end{array}$ \\
\hline Vit. A & $\begin{array}{l}\text { Proteção da córnea. } \\
\text { Indicada para crianças } \\
\text { e gestantes, defesa do } \\
\text { organismo. }{ }^{16}\end{array}$ & $770 \mu \mathrm{g} / \mathrm{dia}^{16}$ & $\begin{array}{l}\text { Leite, carnes, óleos de } \\
\text { peixe, gema de ovo, } \\
\text { folhosos verde-escuro: } \\
\text { amarelos, frutas não } \\
\text { cítricas, oleaginosas. }\end{array}$ \\
\hline Vit. C & $\begin{array}{l}\text { Diminui risco de parto } \\
\text { prematuro, pré-eclâmpsia, } \\
\text { infecções e descolamento } \\
\text { da placenta. }\end{array}$ & $85 \mathrm{mg} / \mathrm{dia}^{13}$ & $\begin{array}{l}\text { Frutas cítricas, } \\
\text { pimentão, agrião. }\end{array}$ \\
\hline Vit. D & $\begin{array}{l}\text { Ganho menor de peso do } \\
\text { concepto - redução da } \\
\text { mineralização óssea. }{ }^{22}\end{array}$ & $15 \mu \mathrm{g} / \mathrm{dia}^{13}$ & $\begin{array}{l}\text { Óleo de fígado de } \\
\text { bacalhau, peixes, } \\
\text { gema de ovo e leite. }\end{array}$ \\
\hline
\end{tabular}




\begin{tabular}{|c|c|c|c|}
\hline Elemento & Função & $\begin{array}{c}\text { Recomendação } \\
\text { nutricional }\end{array}$ & Fonte nutricional \\
\hline Vit. E & $\begin{array}{l}\text { Ação anti-inflamatória e } \\
\text { antioxidante. }\end{array}$ & $15 \mathrm{mg} / \mathrm{dia}^{13}$ & $\begin{array}{l}\text { Gérmen de trigo; } \\
\text { óleos vegetais; } \\
\text { castanhas, gema, } \\
\text { folhosos verdes- } \\
\text { escuros, legumes. }\end{array}$ \\
\hline Vit. K & $\begin{array}{l}\text { Evita hemorragia no } \\
\text { lactente. }{ }^{17}\end{array}$ & $\begin{array}{l}\text { Neonato de } 1,0 \text { a } 2,0 \mathrm{mg} \\
\text { imediatamente após o } \\
\text { parto }\end{array}$ & $\begin{array}{l}\text { Folhosos verde- } \\
\text { escuro, óleo, } \\
\text { oleaginosas, frutas. }\end{array}$ \\
\hline Riboflavina B2 & $\mathrm{ND}^{17}$ & $1,4 \mathrm{mg} / \mathrm{dia}^{13}$ & Carnes, ovos e cereais \\
\hline Folato & $\begin{array}{l}\text { Previne anemias, doenças } \\
\text { cardiovasculares e } \\
\text { neurais. }\end{array}$ & $600 \mu \mathrm{g} / \mathrm{dia}^{13}$ & $\begin{array}{l}\text { Folhosos verdes, } \\
\text { levedo de cerveja, } \\
\text { cenoura, gema de ovo } \\
\text { fígado, feijão, laranja, } \\
\text { banana, beterraba. }\end{array}$ \\
\hline Vit. B12 & $\mathrm{ND}^{17}$ & $2,6 \mu \mathrm{g} / \mathrm{dia}^{13}$ & $\begin{array}{l}\text { Alimentos de origem } \\
\text { animal. }\end{array}$ \\
\hline Tiamina & $\mathrm{ND}^{17}$ & $1,4 \mathrm{mg} / \mathrm{dia}^{13}$ & $\begin{array}{l}\text { Cereais integrais, } \\
\text { nozes, leguminosas, } \\
\text { carne de porco magra }\end{array}$ \\
\hline Niacina B3 & $\mathrm{ND}^{17}$ & $18 \mathrm{mg} / \mathrm{dia}^{13}$ & $\begin{array}{l}\text { Carnes, ovos, farelos, } \\
\text { amendoim, legumes, } \\
\text { abacates e cereais. }\end{array}$ \\
\hline $\begin{array}{l}\text { Ácido } \\
\text { Pantotênico } \\
\text { (B5) }\end{array}$ & $\mathrm{ND}^{17}$ & & $\begin{array}{l}\text { Carne, leite, peixe, } \\
\text { leguminosas, cereais } \\
\text { integrais, vegetais de } \\
\text { folhas verdes, farelo } \\
\text { de trigo, queijo. }\end{array}$ \\
\hline $\begin{array}{l}\text { Piridoxina Vit. } \\
\text { B6 }\end{array}$ & $\mathrm{ND}^{17}$ & $1,9 \mathrm{mg} / \mathrm{dia}^{13}$ & $\begin{array}{l}\text { Carnes, grãos } \\
\text { integrais, vegetais, } \\
\text { gema de ovo e } \\
\text { sementes. }\end{array}$ \\
\hline Cálcio & Formação óssea. & $1000 \mathrm{mg} / \mathrm{dia}^{13}$ & $\begin{array}{l}\text { Carnes, leite e } \\
\text { derivados e vegetais } \\
\text { folhosos verdes- } \\
\text { escuros. }\end{array}$ \\
\hline Ferro & $\begin{array}{l}\text { Reduz o nascimento } \\
\text { de bebês prematuros e } \\
\text { baixo peso e o risco de } \\
\text { morte materna no parto } \\
\text { e no pós-parto imediato; } \\
\text { previne infecções. }{ }^{17}\end{array}$ & $27 \mathrm{mg} / \mathrm{dia}^{13}$ & $\begin{array}{l}\text { Carnes de boi (fígado } \\
\text { e vísceras), carnes } \\
\text { de aves e peixes, } \\
\text { mariscos crus, feijões, } \\
\text { grãos integrais, nozes } \\
\text { e castanhas, melado } \\
\text { de cana-de-açúcar, } \\
\text { rapadura e açúcar } \\
\text { mascavo. }\end{array}$ \\
\hline
\end{tabular}




\begin{tabular}{l|l|l|l}
\hline \multicolumn{1}{c|}{ Elemento } & \multicolumn{1}{|c|}{ Função } & \multicolumn{1}{|c|}{$\begin{array}{l}\text { Recomendação } \\
\text { nutricional }\end{array}$} & \multicolumn{1}{|c}{ Fonte nutricional } \\
\hline Zinco & $\begin{array}{l}\text { Deficiência leva ao } \\
\text { aborto, retardo do } \\
\text { crescimento intrauterino, } \\
\text { prematuridade e pré- } \\
\text { eclâmpsia }\end{array}$ & & $\begin{array}{l}\text { Oleaginosas, carne, } \\
\text { cereais integrais, } \\
\text { semente de abóbora, } \\
\text { feijão e leite. }\end{array}$ \\
\hline
\end{tabular}

$\mathrm{ND}$ - não interfere na gestação

Para gestantes entre 19 anos e 50 anos de idade, ou seja, mulheres adultas, temos o seguinte cálculo do EER (Necessidade Estimada de Energia) apresentado na Tabela 2.

Tabela 2. Cálculo do EER.

\begin{tabular}{l|l}
\hline EER $=$ EER & $+(8$ Kcal $\times$ IG em semanas $)+180 \mathrm{Kcal}$, onde: \\
\hline Pré-gestacional $=354-(6,91 \mathrm{x}$ idade $)+$ PA x $(10 \times$ Peso Kg $)+(934 \times$ altura metros $)+25$ Kcal
\end{tabular}

Dados adaptados de Dal Bosco, SM; 2010. ${ }^{15} \mathrm{PA}=1,0$ (sedentária), $\mathrm{PA}=1,16$ (pouco ativa), $\mathrm{PA}=1,31$ (ativa), $\mathrm{PA}=1,56$ (muito ativa)

O ganho de peso da gestante esperado no primeiro trimestre não se altera em relação ao seu peso corporal inicial, podendo apresentar variações aceitáveis de aumento de até 2 $\mathrm{Kg}$ ou diminuição de até $3 \mathrm{Kg}$. No período entre o segundo e terceiro trimestre o ganho de peso recomendado fundamenta-se no IMC (Índice de Massa Corporal) pré-gestacional. Para o IMC gestacional, deve-se considerar os pontos de corte de forma diferente daqueles adotados para a população adulta padrão (Tabela 3 ). ${ }^{15}$

Tabela 3. Classificação do IMC Pré-Gestacional com o Ganho de Peso (g/semana) e o Ganho Total (Kg)

\begin{tabular}{l|l|l}
\hline IMC Pré-Gestacional & Ganho de Peso $(\mathrm{g} / \mathrm{semana})$ & Ganho Total $(\mathrm{kg})$ \\
\hline Baixo peso $(<19,8)$ & $500 \mathrm{~g}^{*}$ & 12,5 a 18 \\
\hline Normalidade (19,8 a 26$)$ & $400 \mathrm{~g}^{\star}$ & 11,5 a 16 \\
\hline Sobrepeso $(>26$ a 29$)$ & $300 \mathrm{~g}^{\star}$ & 7 a 11,5 \\
\hline Obesidade $(>29)$ & $200 \mathrm{~g}^{\star}$ & 7 a 9,1 \\
\hline
\end{tabular}

Adaptado de IOM $(1992)^{19 *}$ a partir do $2^{\circ}$. trimestre

Classificação do estado nutricional para cada semana gestacional de gestantes gemelares, apresentado na Tabela 4. 
Tabela 6. Classificação do IMC Meta de Perda de Peso Recomendada

\begin{tabular}{l|l|l}
\hline \multicolumn{1}{c|}{ IMC } & \multicolumn{1}{|c}{ Meta } & $\begin{array}{c}\text { Perda de } \\
\text { Peso }\end{array}$ \\
\hline$<18,5$ (baixo peso) & IMC saudável (eutrofia) & \\
\hline$\geq 18,5$ e $<25$ (eutrofia) & Manutenção do peso dentro da eutrofia & \\
\hline$\geq 25$ e $<30$ (sobrepeso) & Perda de peso até atingir IMC eutrófico & $\begin{array}{l}0,5 \mathrm{a} \\
1 \mathrm{Kg} / \mathrm{mês}\end{array}$ \\
\hline$\geq 30$ (obesidade) & Perda de peso até atingir IMC eutrófico & $\begin{array}{l}0,5 \mathrm{a} \\
2 \mathrm{Kg} / \mathrm{mês}\end{array}$ \\
\hline
\end{tabular}

Dados adaptados de Dal Bosco, SM; $2010^{15}$

CONSEQUÊNCIAS DE HÁBITOS ALIMENTARES NÃO ADEQUADOS NA GESTAÇÃO

O desenvolvimento ou programação fetal está relacionado a adversidades intrauterinas que levam a alterações metabólicas, endócrinas e imunológicas permanentes, tornando-se mais evidente na fase adulta. Acredita-se que há um período crítico do desenvolvimento intrauterino onde o embrião e o feto são mais sensíveis às informações provenientes do ambiente. Assim, o concepto prepara-se para viver em um ambiente não favorável após o nascimento, e, em virtude disso, o seu organismo sofre adaptações utilizando esses parâmetros ambientais. Os períodos mais prematuros e finais da gestação são caracterizados pelo amadurecimento do sistema do eixo hipotálamo-hipófise-adrenal e, portanto, estes também são os momentos de maior susceptibilidade do concepto a alterações endócrinas. ${ }^{22}$ Enquanto que, o amadurecimento do metabolismo glicídico ocorre nos períodos finais da gestação, sendo este mais susceptível a alterações na sensibilidade à insulina e tolerância à glicose..$^{23}$ Ambientes intrauterinos adversos podem ocorrer devido a uma variedade de situações, como má nutrição ou excesso nutricional materno, doenças e estresse psicológico. ${ }^{24}$

\section{DEFICIÊNCIA NUTRICIONAL}

Uma das alterações mais comuns no nascimento é a redução de peso do neonato. Nesse caso, o feto, provavelmente, reduz o seu peso, por reorganizar a energia disponível para processos vitais. ${ }^{25} \mathrm{O}$ estudo clássico de coorte com pessoas que viveram em Leningrado o período de fome pela Segunda Guerra Mundial (1941 a 1944) exemplifica o fenótipo econômico (thrifty phenotype), onde a má nutrição materna durante a gestação leva o feto a desencadear alterações no metabolismo energético pressupondo o ambiente malnutrido que ele encontrará após o nascimento. ${ }^{26} \mathrm{Ou}$ tro exemplo típico refere-se à Índia, onde o número de bebês que nascem com peso entre 2,6 e 2,7 kg é o mais comum, o que, provavelmente, tem ligação com a adaptação ao fenótipo econômico desencadeado pela má nutrição materna. Isso corrobora com epidemias de adultos com diabetes tipo II e doenças coronarianas. ${ }^{27,28}$ 


\section{EXCESSO NUTRICIONAL}

No último trimestre da gravidez, os hábitos nutricionais maternos estão relacionados à composição corporal do feto em desenvolvimento; no primeiro ano de vida, que recebe influência direta do aleitamento materno e no crescimento na adolescência, cuja fase de estiramento está ligado aos hábitos alimentares e de atividade física do adolescente, representam os três períodos críticos relacionados à hiperplasia do tecido adiposo, o que dificulta a perda de peso aumentando a tendência à obesidade em adultos. De forma oposta, a obesidade hipertrófica se manifesta ao longo de qualquer fase da vida adulta e está relacionada ao aumento do volume das células adiposas. ${ }^{25}$

\section{QUALIDAde Do LEITE MATERNO}

$\mathrm{O}$ ato de amamentar representa um vínculo entre mãe e filho, troca de carinho e afeto e deve ser administrado de forma livre, o que aprimora o desenvolvimento psicológico, imunológico e neurológico do neonato No Brasil, o aleitamento materno é incentivado pelos órgãos federais, estaduais e municipais por estar relacionado também ao aspecto econômico. ${ }^{15}$

Dados antropométricos da mãe não têm relação com o volume de leite produzido. $\mathrm{O}$ consumo diferenciado de macronutrientes não interfere na constituição do leite materno, com exceção dos lipídios, pois a quantidade e a qualidade da gordura da dieta materna afetam o teor de lipídeos totais do leite, assim como o de ácidos graxos importantes como o ácido docosahexanóico e ácido araquidônico. Já o consumo ade- quado de micronutrientes é essencial para o desenvolvimento do neonato e o conteúdo destes no leite está diretamente relacionado à dieta materna. ${ }^{21}$

Os elementos imunológicos presentes no leite materno são, principalmente, os da família IgA, que são preservados no trato digestório e absorvidos integralmente pelo intestino delgado do neonato. Citocinas e fatores inflamatórios também são compartilhados do organismo materno para o filho por meio da lactação. As principais citocinas presentes no leite materno de mães com quadro de hipersensibilidade são Interleucinas -4, -5, -13 e TGF (Fator de Crescimento Tumoral). Porém, estas estão diminuídas em mães saudáveis. Além desses fatores imunológicos, a presença de diferentes ácidos graxos pode conferir maior imunoproteção do que outros. ${ }^{28} \mathrm{O}$ ácido araquidônico, por exemplo, está mais relacionado ao desenvolvimento de resposta de hipersensibilidade do que o ácido eicospentanóico. ${ }^{29} \mathrm{~A}$ dieta recomendada para a lactante deve conter quantidades suficientes de ácidos graxos poli-insaturados de cadeia longa (AGPI-CL), que atendem às necessidades maternas e do bebê, agindo como um elemento protetor contra alergia e infecções. ${ }^{28}$

Em relação a outras doenças, foi realizada uma revisão sistemática entre o período de 1996 a 2006 para avaliar os efeitos da amamentação sobre a pressão arterial, diabetes, colesterolemia, sobrepeso, obesidade e desempenho intelectual. Assim, verificou-se uma redução na pressão arterial, no colesterol total plasmático, sobrepeso, obesidade e Diabetes tipo 2, e melhor desempenho nos testes de inteligência dos neonatos. ${ }^{30}$

Outro estudo realizado com 134 pré-esco- 
lares entre 3 e 5 anos de idade de uma escola particular de Brasília associou o tempo de amamentação exclusiva à adiposidade central e periférica. Foi sugerido que crianças amamentadas exclusivamente até os seis meses possuíam uma menor tendência à ocorrência de sobrepeso e obesidade, como também, uma correlação inversa significativa entre perímetro da cintura e tempo de amamentação. ${ }^{31}$

\section{ProgramaÇão fetal: doen- ÇAS CRÔNICAS PROGRAMADAS PELA NUTRIÇÃO MATERNA}

O termo "Programação Fetal" foi utilizado por Hales e Barker ${ }^{6}$ para demonstrar que doenças metabólicas têm sua origem já nas primeiras experiências nutricionais durante a gestação e a lactação. Um estresse ou um estímulo nutricional durante o período de desenvolvimento fetal resultam em respostas adaptativas do feto (programação metabólica) que são vantajosas para a sua sobrevivência em um ambiente com condições abaixo do ideal. ${ }^{7}$

Em animais, verificou-se que a dieta hiperlipídica materna está associada nos filhotes a um fenótipo semelhante à Síndrome Metabólica humana. Estudos mostram que, pelo menos em parte, a Síndrome Metabólica em adultos pode ter suas origens no período fetal ou na infância. A partir da terceira semana do desenvolvimento fetal, a sensibilidade embrionária é maior aos efeitos ambientais, por causa dos hormônios embrionários. Sendo um período crítico para teratogênicos.

O consumo prolongado de dieta hiperlipídica por ratas fêmeas acarreta nos filhotes machos maior peso corporal, aumento nos níveis plasmáticos de insulina, glicose, ácidos graxos livres, triglicérides e intolerância à glicose. ${ }^{3}$

Estudos em humanos e animais sugerem que a nutrição materna pode induzir o desenvolvimento programado de hipertensão na prole adulta. ${ }^{31} \mathrm{E}$ a administração de dieta hiperlipídica, às mães, causa na prole adulta: aumento da pressão sanguínea, resistência à insulina, dislipidemia e obesidade. ${ }^{32}$

\section{CONSIDERAÇõES FINAIS}

A qualidade da alimentação da gestante e da lactante está diretamente ligada ao desenvolvimento da prole e à prevalência de doenças crônicas na fase adulta dos descendentes. A subnutrição e o excesso nutricional materno aumentam a susceptibilidade dos filhos a alterações metabólicas, neuronais e no crescimento. Dessa forma, a atenção com a nutrição materna deve ser mais valorizada e discutida, divulgando de maneira mais ampla a importância de uma alimentação saudável nesses períodos críticos do desenvolvimento.

\section{REFERÊNCIAS}

1 Gottlieb MGV, Cruz IBM, Bodanese LC. Origem da síndrome metabólica: aspectos genético-evolutivos e nutricionais. Scientia Medica 2008; 18(1): 31-8.

2. Costa-Silva, J. H., Simões-Alves, A. C., Fernandes, M. P. Developmental Origins of Cardiometabolic Diseases: Role of the Maternal Diet. Frontiers in Physiology, November 2016, vol. 7. 
3 Roza, NAV., Possignolo L. F., Palanch A. C., Gontijo J. A. Effect of long-term high-fat diet intake on peripheral insulin sensibility, blood pressure, and renal function in female rats. Food Nutr Res. 2016, feb 12; 60: 28536.

4 Hales CN \& Barker D J. The thrifty phenotype hypothesis. Br Med Bull 2001; 60: 5-20.

5 Barker DJP. Fetal origins of coronary heart disease. Br Med 1995; 311: 171-4.

6 Lowensohn, R. I., Stadler, D. D., Naze, C. Current Concepts of Maternal Nutrition. Obstetrical and Gynecological Survey, 2016, vol. 71, n. 7.

7 Chang GO, Gaysinskaya V., Karatayev O., Leibowitz SF. Maternal High-Fat Diet and Fetal Programming: Increased Proliferation of Hypothalamic Peptide-Producing Neurons That Increase Risk for Overeating and Obesity. J Neurosc. 2008; 28(46): 12.107-119.

8 Belarmino GO, Moura ERF, Oliveira NC, Freitas GL. Risco nutricional entre gestantes adolescentes. Acta Paulista Enferm. 2009; 22(2): 169-75.

9 Duffy EM, Bonham MP, Wallace JMW, Chang CK, Robson PJ, Myers GJ, et al. Iron status in pregnant women in the republic of Seychelles. Public Health Nutr. 2009; 13(3): 331-7.

10 Kalaivani K. Prevalence \& consequences of anaemia in pregnancy. Indian J Med Res. 2009; 130: 627-33.

11 Viana JML, Tsunechiro MA, Bonadio I., Fujimori E. Adequação do consumo de ferro por gestantes e mulheres em idade fértil atendidas em um serviço de pré-natal. Mundo Saúde. 2009; 33(3): 286-93 (Belarmino).

12 Godfrey, K. M., Costello, P., Lillycrop, K. Development, epigenetics and metabolic programming, Nestle Nutr Inst Workshop Ser. 2016; 85: 71-80.

13 Scholl TO, Hediger ML, Ances IG. Maternal growth during pregnancy and decreased infant birth weight. Am J Clin Nutr 1990; 51(5): 790-3.

14 Dietary Reference Intakes: Applications in Dietary Assessment 2000 [acesso 05/jun./2014]; disponível <http://www.nap.edu/search/?term=Dietary+Reference+Intakes $>$

15 Dal Bosco S., Conde SR. Nutrição e Saúde,1. ed. Lajeado: Ed. Univates; 2013.

16 BRASIL. Programa Nacional de Alimentação e Nutrição, 2011 [acesso 05/jun./2014]; disponível $<$ http://dab.saude.gov.br/portaldab/pnan.php>

17 Parizzi MR, Fonseca JGM. Nutrição na gravidez e na lactação. Rev Med Minas Gerais 2010; 20(3): 341-53.

18 Vitolo, MR. Nutrição - Da Gestação ao Envelhecimento, 1. ed. Rio de Janeiro: Ed. Rubio; 2008.

19 INSTITUTE OF MEDICINE - IOM. Nutrition during pregnancy and lactation: an implementation guide. Washington, DC: National Academic Press, 1992.

20 Luke B., Hediger ML, Nugeht C., Newman RB, Mauldin JG, Witter FR, et al. Body mass indexspecific weight gains associated with optimal birth weights and twin pregnancies. J Reprod Med. 2003; 48(4): 217-24.

21 Accioly, ES; Lacerda C.; Aquino EM. Nutrição em Obstetrícia e Pediatria, 2. ed. São Paulo: Ed: Guanabara Koogan, 2009.

22 Fisher RE, Karrow NA, Quinton M, et al. Endotoxin exposure during late pregnancy alters ovine offspring febrile and hypothalamic-pituitary-adrenal axis responsiveness later in life, Stress 2010; 13(4): 334-42.

23 Ravelli ACJ, Van Der Meulen JHP, Osmond C, Barker DJP, O. P. Bleker OP. Obesity at the age of $50 \mathrm{y}$ in men and women exposed to famine prenatally. American Journal of Clinical Nutrition. 1999; 70(5): 811-16.

24 Fisher RE, Steele M., and Karrow NA. Fetal Programming of the Neuroendocrine-Immune System and Metabolic Disease. J Pregnancy. 2012; 2012: 1-10. 
25 McARDLE WD, KATCH FI, KATCH VL. Fisiologia do Exercício, 5. ed. Ed Guanabara Koogan, 2003.

26 Burke K. T., Colvin P. L., Myatt L., Graf G. A., Schroeder F., Woollett L. A. Transport of maternal cholesterol to the fetus is affected by maternal plasma cholesterol concentrations in the golden syrian hamster. J Lipid Res. 2009; 50(6): 1.146-55.

27 Wang, K. C. W., Botting, K. J., Zhang, S., McMillen, I C., Brooks, D. A., Morrison, J. L. Akt signaling as a mediator of cardiac adaptation to low birth weight. Journal of Endocrinology, 2017, 233, R81-R94.

28 Tinoco SMB, Sichieri R., Moura AS, Santos FS, Carmo MGT. Importância dos ácidos graxos essenciais e os efeitos dos ácidos graxos trans do leite materno para o desenvolvimento fetal e neonatal. Cad. Saúde Pública. 2007; 23(3): 525-34.

29 Horta BL, Bahl R., Martines JC, Victora CG. Evidence on the long-term effects of breastfeeding Systematic Reviews and meta-analyses. World Health Organization. 2007. Disponível: <http:// whqlibdoc.who.int/publications/2007/9789241595230_eng.pdf>.

30 Moraes JFVN, Giugliano R. Aleitamento materno exclusivo e adiposidade. Rev Paul Pediatr, 2011; 29(2): 152-6.

31 Rasyid, H., Bakri, S. Intra-uterine Growth Retardation and Development of Hypertension. Acta Medica Indonesiana, 2016, vol. 48 • Number 4.

32. Howell, K. R., Powell, T. L. Effects of maternal obesity on placental function and fetal development. Reproduction, 2017, 153 R97-R108.

\section{Dados das Autoras}

\section{Carolina Bellato de Souza Campos}

Graduada em Nutrição pela Universidade Metodista de Piracicaba. Piracicaba/SP - Brasil. carolinabellato@hotmail.com

\section{Adrianne Christine Palanch}

Doutora em Ciências pela Universidade de São Paulo. Professora da Faculdade de Ciências da Saúde da Universidade Metodista de Piracicaba. Piracicaba/SP - Brasil.

apalanch@unimep.br

Submetido em: 5-6-2014

Aceito em: 18-4-2017 